This is an electronic reprint of the original article. This reprint may differ from the original in pagination and typographic detail.

Author(s): Witulski, A. F.; Arslanbekov, R.; Raman, A.; Schrimpf, R. D.; Sternberg, A.; Galloway, K. F.; Javanainen, Arto; Grider, D.; Lichtenwalner, D. J.; Hull, B.

Title: $\quad$ Single Event Burnout of SiC Junction Barrier Schottky Diode High-Voltage Power Devices

Year: $\quad 2018$

Version:

Please cite the original version:

Witulski, A. F., Arslanbekov, R., Raman, A., Schrimpf, R. D., Sternberg, A., Galloway, K. F., Javanainen, A., Grider, D., Lichtenwalner, D. J., \& Hull, B. (2018). Single Event Burnout of SiC Junction Barrier Schottky Diode High-Voltage Power Devices. IEEE Transactions on Nuclear Science, 65(1), 256-261.

https://doi.org/10.1109/TNS.2017.2782227

All material supplied via JYX is protected by copyright and other intellectual property rights, and duplication or sale of all or part of any of the repository collections is not permitted, except that material may be duplicated by you for your research use or educational purposes in electronic or print form. You must obtain permission for any other use. Electronic or print copies may not be offered, whether for sale or otherwise to anyone who is not an authorised user. 


\title{
Single Event Burnout of SiC Junction Barrier Schottky Diode High-Voltage Power Devices
}

\author{
A. F. Witulski, Senior Member, IEEE, R. Arslanbekov, A. Raman, Member, IEEE, \\ R. D. Schrimpf, Fellow, IEEE, A. Sternberg, K.F. Galloway, Life Fellow, IEEE, \\ A. Javanainen, Member, IEEE, D. Grider, D. J. Lichtenwalner, B. Hull
}

\begin{abstract}
Ion-induced degradation and catastrophic failures in high-voltage SiC Junction Barrier Schottky (JBS) power diodes are investigated. Experimental results agree with earlier data showing discrete jumps in leakage current for individual ions, and show that the boundary between leakage current degradation and a single-event-burnout-like effect is a strong function of LET and reverse bias. TCAD simulations show high localized electric fields under the Schottky junction, and high temperatures generated directly under the Schottky contact, consistent with the hypothesis that the ion energy causes eutectic-like intermixture at the metal- semiconductor interface or localized melting of the silicon carbide lattice.
\end{abstract}

Index Terms - Single event effects, heavy ions, silicon carbide, power diodes, junction barrier schottky (JBS) diode, single-event burnout, thermal coefficients of silicon carbide.

\section{INTRODUCTION}

Silicon carbide ( $\mathrm{SiC}$ ) has excellent properties for use in power device applications. In comparison to silicon, silicon carbide has a higher critical electric field and thermal conductivity. In addition, efficient Schottky switching diodes in $\mathrm{SiC}$ can be manufactured that exhibit very low on-state voltage and minimal turn-off switching loss with almost no reverserecovery behavior, which makes $\mathrm{SiC}$ a good candidate for space power conversion applications. However, the sensitivity of $\mathrm{SiC}$ power devices (MOSFETs and diodes) to particle radiation has been found to be higher than expected, given the wide bandgap and high critical electric field, as shown by multiple researchers who have consistently measured significant leakage current increases and single-event burnout in $\mathrm{SiC}$ devices [1-11].

Manuscript submitted July 12, 2017, revised Nov. 18 and Nov. 24, 2017, accepted Dec. 4, 2017. Support for this work provided by NASA under SBIR Contract No. NNX16CG55P and ESI Grant No: NNX17AD09G.

University of Jyväskylä was supported by ESA/ESTEC Contract No. 4000111630/14/NL/PA and the Academy of Finland Centre of Excellence Programme 2012-2017 Project No. 2513553.

A. Sternberg, K.F. Galloway, A. Javanainen, R. D. Schrimpf, A. F. Witulski are with Vanderbilt University, Department of Electrical Engineering and Computer Science, VU Station B 351824, 2301 Vanderbilt Place, Nashville, TN 37235, USA; (615) 322-2771, Fax: (615) 343-6702.

A. Javanainen is with the University of Jyvaskyla, Department of Physics, P.O. Box 35, FI-40014, University of Jyvaskyla, Finland.

A. Raman and R. Arslanbekov are with CFD Research Corporation (CFDRC), 701 McMillian Way, Suite D, Huntsville, AL 35806.

D. Grider, D. J. Lichtenwalner, and B. Hull are with Wolfspeed, a Cree Company, 3028 E. Cornwallis Road, RTP, NC 27709.
There are several examples of SiC power diode SEB in the literature [1-7]. $\mathrm{SiC}$ diodes show three regions of response as a function of reverse bias and the parameters of heavy-ion exposure: 1) non-destructive; 2) gradual degradation; and 3) catastrophic failure through Single Event Burnout (SEB). Kuboyama et al. [1] illustrated the three regions of interest when $\mathrm{SiC}$ Schottky diodes are exposed to heavy-ion radiation, as shown in Fig. 1(a). Region 1 is not destructive and the collected charge can be measured reproducibly; in Region 2, there is a permanent leakage current increase; and catastrophic failure occurs in Region 3 (here designated single-event burnout (SEB), although it differs from the bipolar action responsible for SEB in transistors). The anticipated mapping of

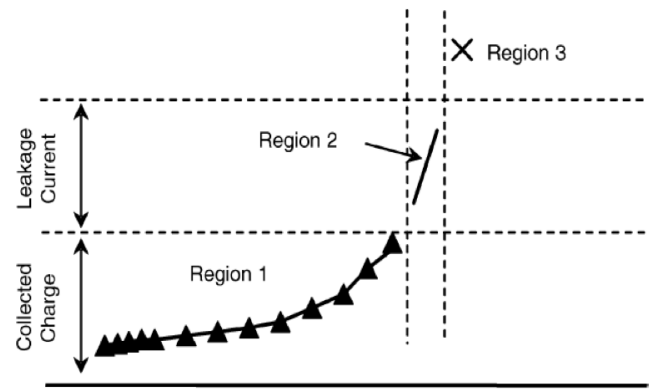

Bias Voltage $[\mathrm{V}]$

(a)

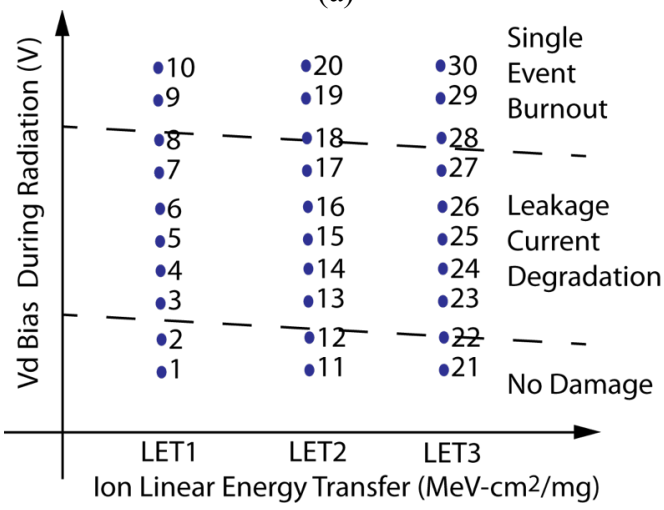

(b)

Fig. 1. (a) Conceptual definition of three degradation regions of $\mathrm{SiC}$ diodes in heavy ions, after [1]. (b) Expected mapping of the three degradation regions of $\mathrm{SiC}$ diodes under heavy-ion irradiation and dependence on test variables. Numbers represent pristine devices irradiated at a given LET and off-state bias. 
JBS Anode

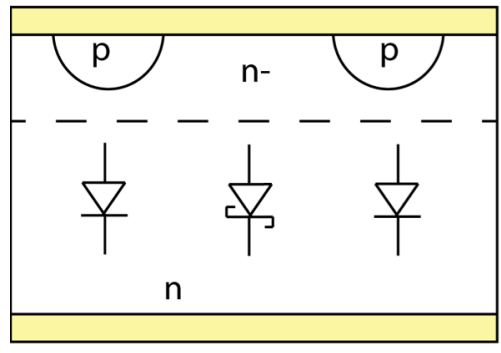

JBS Cathode

Fig. 2. Notional diagram of a junction-barrier Schottky (JBS) diode [13].

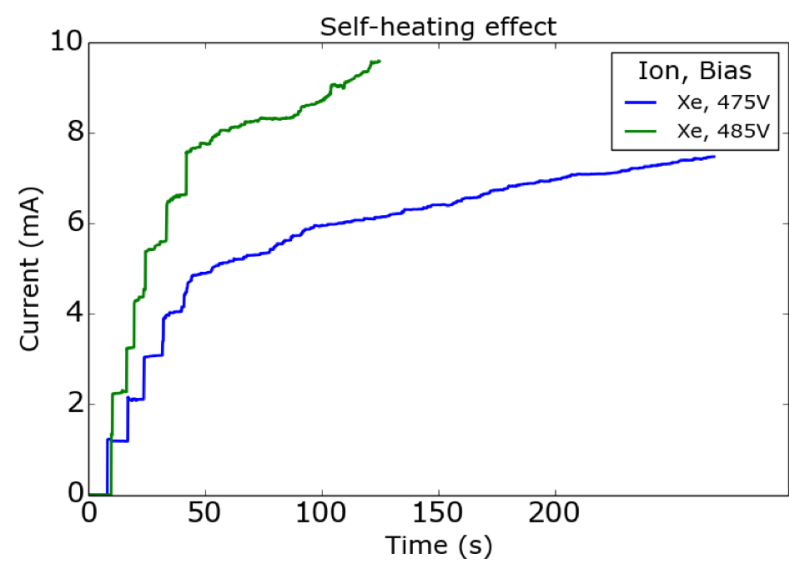

Fig. 3. Measured diode current vs. time during heavy-ion irradiation for two diodes, showing discrete increases in leakage current with individual heavy ions, with selfheating effect. Xenon flux was 68 , Neon was 5.7 to 6.1 ions $/ \mathrm{cm}^{2} / \mathrm{sec}$.

the degradation regions into the test variable space of diode offstate bias, linear energy transfer (LET), and ion fluence is shown in Fig. 1(b).

Many commercial high voltage SiC diodes use the Junction Barrier Schottky (JBS) design [12], as shown in Fig. 2. Regularly spaced $\mathrm{P}+$-wells are added just below the Schottky barrier interface, yielding lower leakage currents, higher breakdown voltages, and increased reliability compared to conventional SiC Schottky Barrier Diodes (SBDs). A depletion region is formed at the interface of the $\mathrm{P}^{+}$-wells and the $\mathrm{N}^{-}$drift layer that in the reverse-bias state extends between the $\mathrm{p}+$ regions and substantially prevents the Schottky interface from conducting current under reverse bias. In the forward conduction state, only the Schottky diode portion conducts under normal operation because the on-voltage of $\mathrm{SiC} \mathrm{P-i-N}$ diodes is high (on the order of $3 \mathrm{~V}$ because of the wide $\mathrm{SiC}$ bandgap, instead $\sim 0.7 \mathrm{~V}$ for $\mathrm{Si}$ ).

Abbate et al. $[7,8]$ presented electro-thermal simulations that indicate ion-generated charge can cause the temperature at the Schottky barrier to become higher than the SiC melting point long enough to cause permanent damage. Additionally, Javanainen et al. $[10,11]$ presented data and simulations that suggest that temperature increases are responsible for the gradual degradation behavior of Region 2 of Fig. 1 .

In this paper, experimental data on both leakage current degradation and catastrophic failure in $\mathrm{SiC}$ Junction Barrier Schottky (JBS) diodes are presented. The dependence of the threshold for leakage degradation and the threshold for SEB are mapped as functions of bias and LET. The experimental results are interpreted based on TCAD simulations. We suggest that at low ion LET and off-state bias, a somewhat lower temperature in the ion deposition region $(\sim 1000 \mathrm{~K})$ causes intermixture of the metal and semiconductor at the Schottky interface, which increases the leakage current. At high LET and off-state bias, the energy deposited is sufficient to raise the temperature to the melting point of SiC (variously reported as 2900-3100 K), resulting in a catastrophic burnout event.

\section{HEAVY-ION EXPERIMENTAL RESULTS FOR JBS SiC DEGRADATION AND SEB}

Twenty-five Wolfspeed C4D020120A (1200 V, 20 A) silicon carbide diodes in open TO-247 packages were tested. The heavy-ion irradiations were performed at the TAMU Cyclotron using ions from the $15 \mathrm{MeV} / \mathrm{amu}$ cocktail with sufficient range to penetrate the entire die thickness. The ions and initial LETs in air were $\mathrm{Ne}, \mathrm{Ar}, \mathrm{Kr}, \mathrm{Xe}$, with 2.8, 8.0, 27, and $49 \mathrm{MeV}^{*} \mathrm{~cm}^{2} / \mathrm{mg}$, respectively. The variables during the heavy-ion test were the drain-source voltage bias, the flux of the beam, and the energy of the ions. The beam flux was between 5 and 100 ions $/ \mathrm{cm}^{2} / \mathrm{s}$, depending on the test.

Drain leakage current degradation is shown in Fig. 3 for a very low ion flux. Significant discrete increases in leakage current are evident for individual ion strikes, consistent with earlier experiments $[9,10]$. Leakage current increases as long as the beam is on. Initial defects produced at the Schottky barrier cause the biggest changes in the diode blocking capability; later defects make a smaller percentage change in the blocking capability, and appear as smaller steps in the I-V curve. There were no heatsinks used during the ion test.

Drain current versus time plots of single-event burnout events are shown in Fig. 4, showing burnout events for various ions and reverse bias conditions, consistent with observations

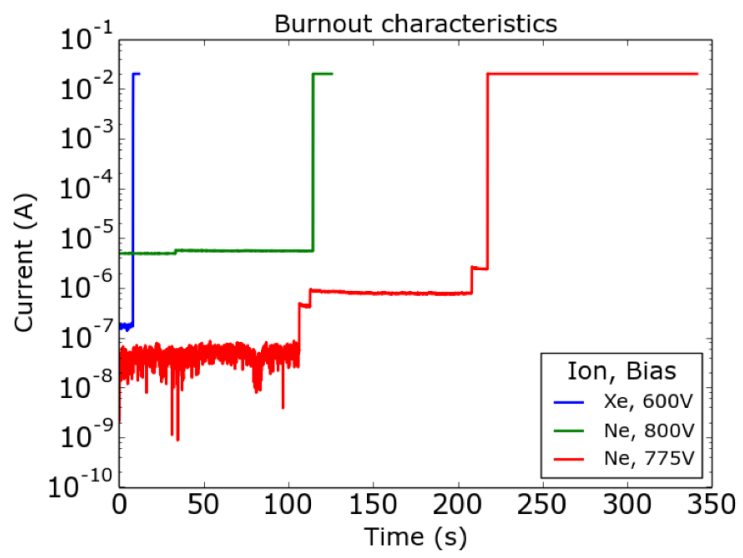

Fig. 4. SEB events for three different devices. Xenon flux was 68 , Neon was 5.7 to $6.1 \mathrm{ions} / \mathrm{cm}^{2} / \mathrm{sec}$. All diodes are irradiated at room temperature. 


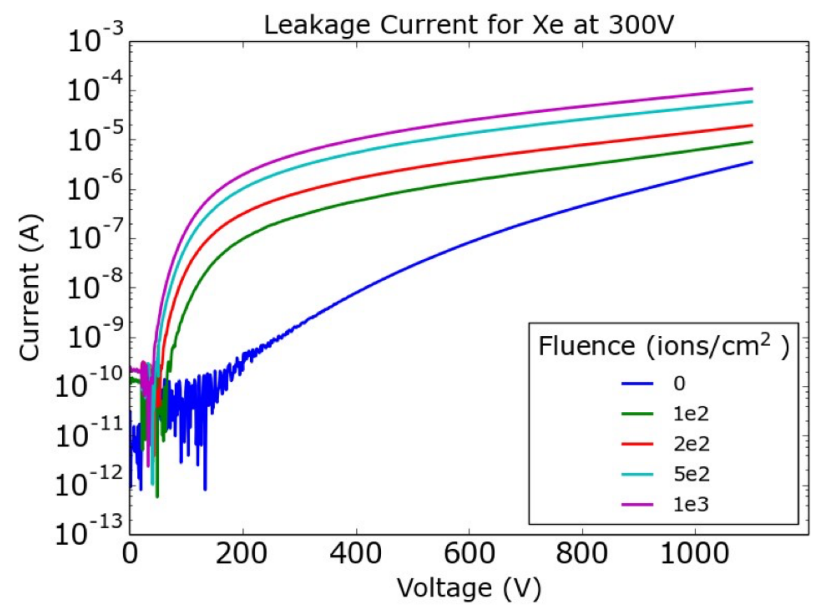

Fig.5 Leakage current vs. voltage for diodes irradiated with $\mathrm{Xe}$ ions while biased at $300 \mathrm{~V}$ at various fluences.

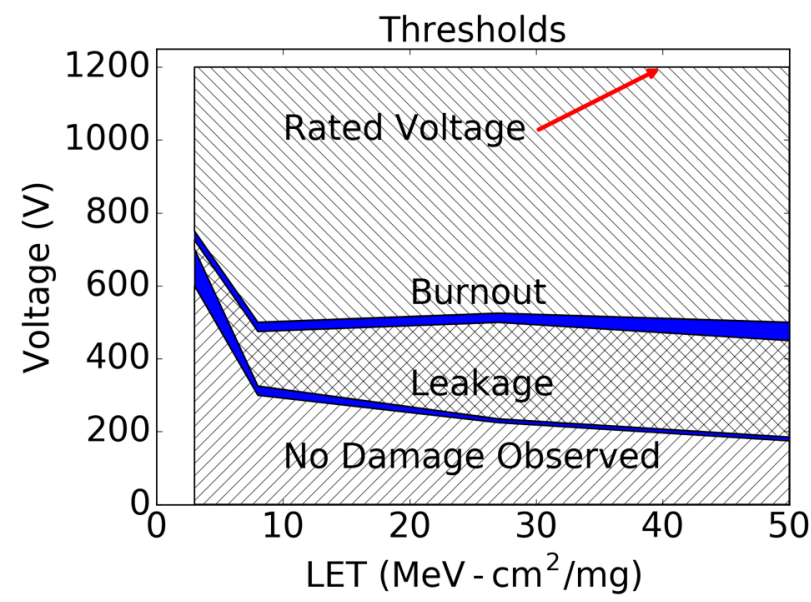

Fig. 6. Measured degradation mode thresholds as functions of applied bias and LET. The width of the line indicates minimum voltage at which burnout was observed and maximum voltage with no burnout observed.

of previous authors [4,5]. Note the very rapid increase of current spanning more than five orders of magnitude.

We investigated the effects of the ion fluence on the diode reverse $I-V$ characteristics. Figure 5 depicts the reverse current vs. voltage after several ion fluences. The devices were biased at $300 \mathrm{~V}$ and placed in a Xe beam, then leakage current was measured as a function of fluence. Measureable damage occurs, even at low fluences. At 100 ions $/ \mathrm{cm}^{2}$, there is a notable increase in the leakage current at lower biases. This trend continues with increasing fluence. At all fluences there is a rapid rise in leakage at low bias $(<200 \mathrm{~V})$, then a more gradual increase of leakage with bias above $200 \mathrm{~V}$. The source-meter used in this experiment had a maximum voltage of $1100 \mathrm{~V}$. However, even with significant damage, as evidenced by the increase in leakage current, the breakdown voltage was still greater than $1100 \mathrm{~V}$.

Figure 6 shows the approximate threshold boundaries for

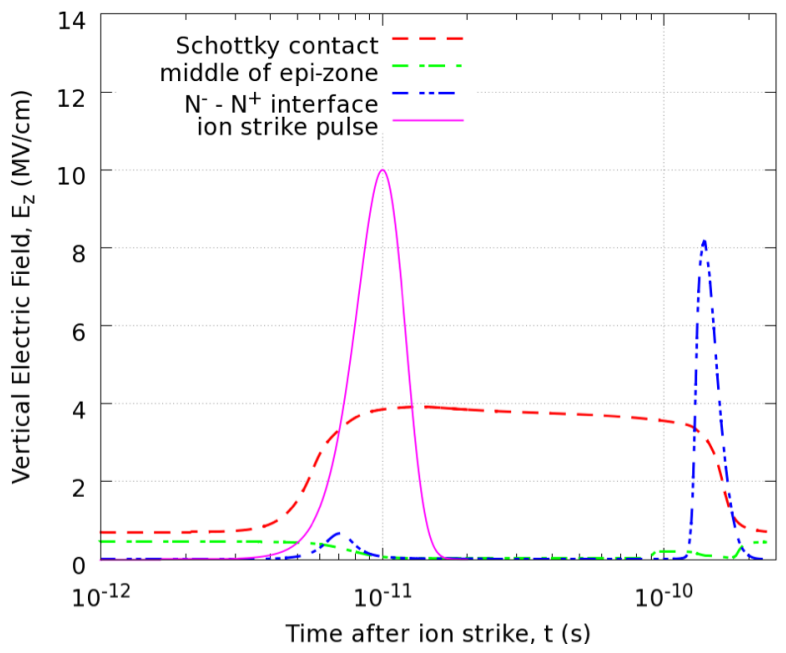

Figure 7. Simulated time evolution of vertical electric field $E_{z}$ at 3 locations for reverse bias of $500 \mathrm{~V}$. The Xe ion strike has LET $=62.4 \mathrm{MeV} /\left(\mathrm{mg} / \mathrm{cm}^{2}\right)$. The ion strike pulse is a notional depiction of the Gaussian charge deposition.

leakage degradation and the threshold boundary for SEB as functions of bias and LET. These boundaries illustrate the three regions of radiation response (Fig. 1(b)) plotted in test-variable space. Previous experiments have not mapped out the dependence of these damage thresholds on bias and LET. Notice the convergence of the leakage current boundary and the SEB boundary at low LET and the relatively high bias voltage needed for an SEB event at low LET. The thresholds have some device-to-device variation, but the largest source of variation in Fig. 6 is due to the granularity of the bias voltages and relatively low number of parts tested. This curve for $\mathrm{SiC}$ diodes shows a nonlinear dependence on LET.

We irradiated one device with Xe ions at a bias of $300 \mathrm{~V}$ to investigate the cumulative effects of leakage current. The $300 \mathrm{~V}$ bias is greater than the damage threshold for Xe, but less than the SEB threshold. After each irradiation step the device was swept to $1100 \mathrm{~V}$ to extract an I/V curve. No evidence of latent damage was observed, and the breakdown voltage of the diode remained higher than the maximum power supply voltage of $1100 \mathrm{~V}$.

\section{Simulation Results For QuASI-3D TCAD MODEL}

A TCAD model of the JBS diode was generated using CFDRC's NanoTCAD simulation software, based on device information provided by Wolfspeed and from the literature [1318].

The NanoTCAD simulator database contains detailed models for the $4 \mathrm{H}-\mathrm{SiC}$ material system such as energy bandgap, carrier effective masses, carrier mobilities, thermal conductivity, incorporating dependencies on temperature, electric field, doping concentrations, and other factors according to $[19,20,21,22]$. In particular, for the electron mobility, the model from Roschke and Schwierz [22] was used, which accounts for dependence on temperature, doping concentration, and saturation under high electric fields. The electron and hole 


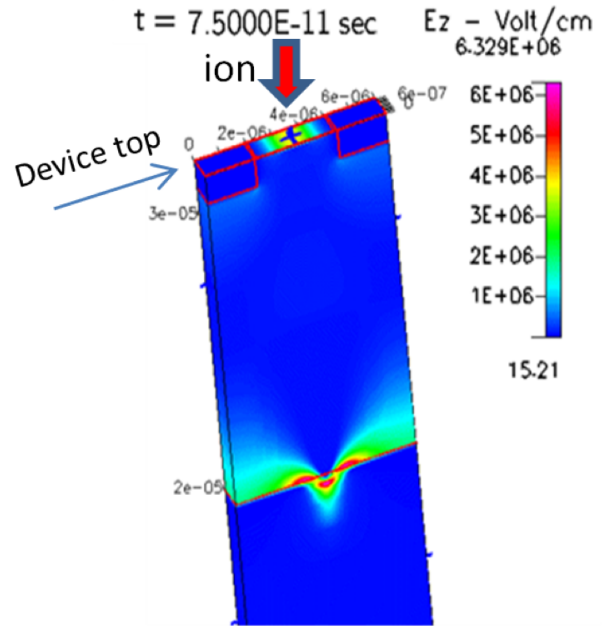

Fig. 8. Simulated spatial electric field contours showing spike (yellow or red) under Schottky contact and epi-sub interface.

lifetimes were assumed to be constant. In the TCAD model, the relationship between the tunneling current density and the electric field is expressed in terms of the surface electric field and the Schottky barrier height, using the Fowler-Nordheim equation [23]. Following [23], the theoretical tunneling effective mass $m^{*}$ can be calculated as $0.33 m_{0}$ for the current direction along the c-axis based on previously reported effective mass tensor values for $4 \mathrm{H}-\mathrm{SiC}$. Then the values for $A_{T}$ and $B_{T}$ are $3 \times 10^{-6}\left(\mathrm{~A} \cdot \mathrm{eV} / \mathrm{V}^{2}\right)$ and $4.4 \times 10^{7}\left(\mathrm{~V} / \mathrm{cm} \cdot(\mathrm{eV})^{3 / 2}\right)$, respectively, (see also [24] where a different set of these parameters was suggested). These values are within $\sim 40 \%$ deviation from the theoretical values, which is acceptable considering the uncertainty of the electron effective mass at the $\mathrm{SiC}$ surface [23]. In the modeled JBS device, the p-wells are considered as highly doped with a p-doping level of $10^{19} \mathrm{~cm}^{-3}$. The doping level in the lightly doped $\mathrm{N}^{-}$epi layer is a parameter which was varied in the range $N_{D}=(1-8) \times 10^{15} \mathrm{~cm}^{-3}$ while the highly doped $\mathrm{N}^{+}$substrate layer was assumed to have a doping of $\mathrm{N}_{\mathrm{A}}=5 \times 10^{18} \mathrm{~cm}^{-3}$. Presently, the TCAD model does not include treatment of defects and their role in the degradation of leakage current. We are working on adding the effects of defects in the model, e.g., via volume and surface traps. Molecular dynamics (MD) simulations are also planned later to provide further insight into ion-induced damage to the $\mathrm{SiC}$ crystal lattice and resulting defect generation.

The simulated current-voltage characteristics were verified against experimental data. The simulation is performed in a 2D planar slab (width corresponds to ion track radius) to approximate the 3-dimensional behavior of an ion strike. The EHP generation rate in the $\mathrm{SiC}$ due to an ion strike was described by a Gaussian in space and time, with a charge generation maximum at $10 \mathrm{ps}$, a characteristic radius of $0.1 \mu \mathrm{m}$, and a characteristic time of $2 \mathrm{ps}$.

Figure 7 shows the electric field vs. time at different locations along a Xe ion strike for a strike location midway between the JBS junctions. For these simulations, the Xe ion strike is assumed to have LET $=62.4 \mathrm{MeV} /\left(\mathrm{mg} / \mathrm{cm}^{2}\right)$ and total

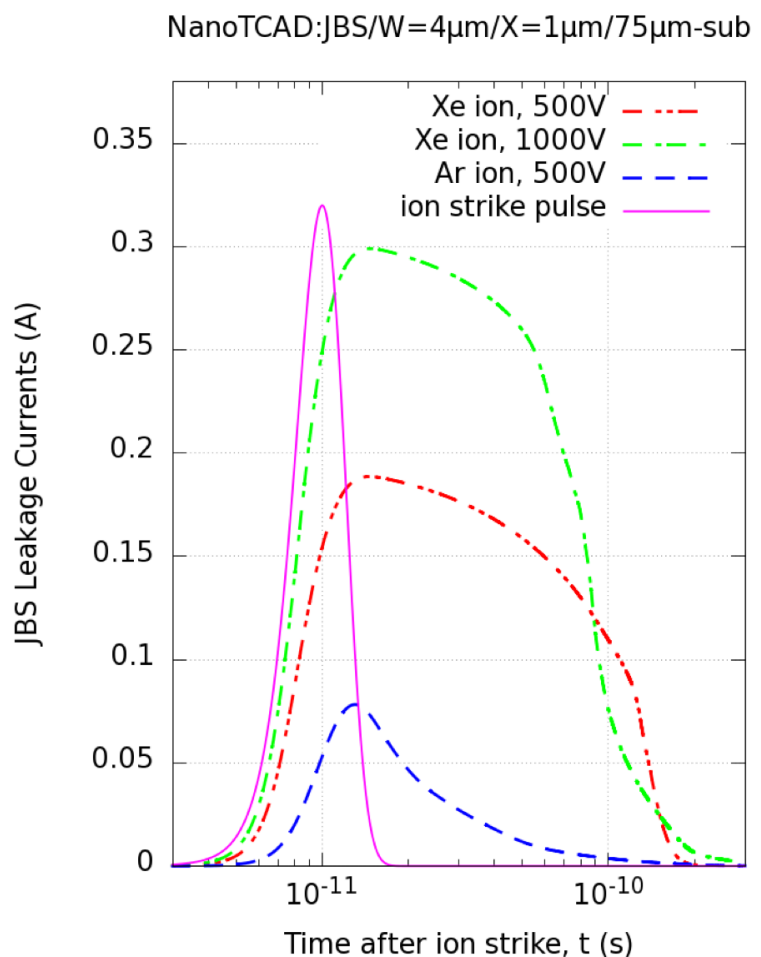

Fig. 9. Simulated ion-induced current in JBS diode at 2 different reverse biases $(500 \mathrm{~V}$ and $1000 \mathrm{~V})$ and 2 ions (Ar, LET 10.9, and Xe, LET 62.4). The ion strike pulse is a notional depiction of the Gaussian charge deposition profile in time.

range $=60 \mu \mathrm{m}(12 \mu \mathrm{m}$ in the epi and $48 \mu \mathrm{m}$ in the substrate layers). Localized electric field maxima occur just below the Schottky contact $(\sim 4 \mathrm{MV} / \mathrm{cm})$ and at the $\mathrm{n}-/ \mathrm{n}+$ epi-substrate interface, where the electric field increases to $\sim 8-10 \mathrm{MV} / \mathrm{cm}$. The first spike is independent of the reverse bias, while the second depends strongly on the reverse bias. The spatial distribution of the electric field at $75 \mathrm{ps}$ after the strike is shown in Fig. 8. The electric field collapses in the epitaxial region due to the high charge density from the strike, and significantly increases near the contact and the epitaxial transition region from low to high doping due to charge pile-up in those two regions.

Figure 9 shows ion-induced current in a JBS diode for two different reverse biases and two different LETs. The peak currents generated are not proportional to the LET, but the greater charge deposition from the high-LET ion results in higher diode terminal current for significantly longer time. On the other hand, for the same ion and different bias voltages, the peak diode reverse current may be roughly proportional to bias voltage.

Coupled electro-thermal simulations were conducted using a three-dimensional implementation of the partial differential heat equation, with appropriate coefficients for silicon carbide. Physical interpretation of "temperature" is imprecise at very small time and distance scales on the order of nanoseconds and nanometers, but the results provide useful insight. The physics of the local energy distribution at those small time and distance 


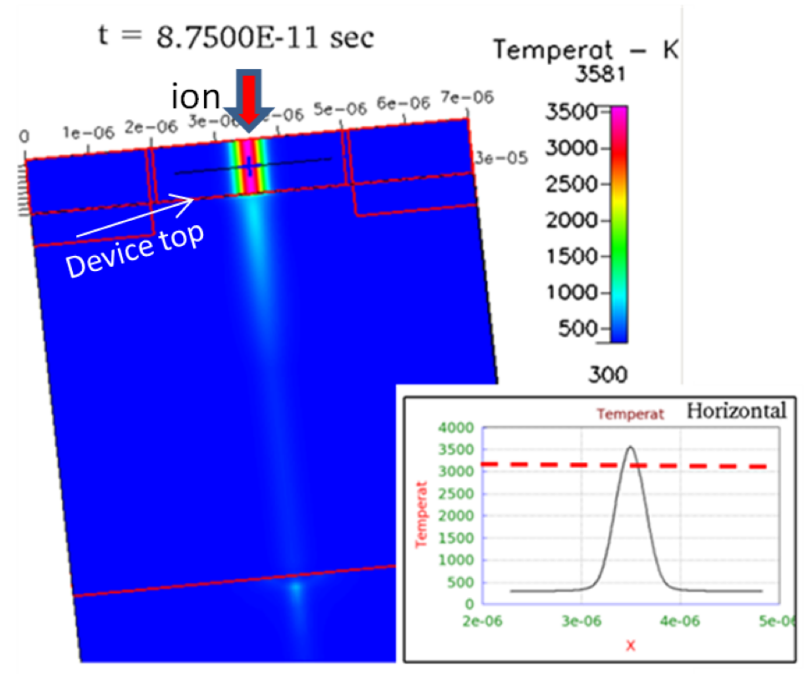

Fig. 10. Temperature contours in the JBS diode showing Xeion induced localized heating profile for ion strike region under the Schottky contact (ion strike located midway between two JBS regions).

scales in non-equilibrium conditions is much more complex than the physics of heat transfer in bulk materials assumed by the conventional heat equation. Furthermore, the semiconductor models used in the TCAD simulator are not verified for use at such high temperatures, for example, the values for electron and hole mobilities in $\mathrm{SiC}$ are experimentally validated only for temperatures up to about $700 \mathrm{~K}$ [25]. However, the computation of the local temperature by means of the heat equation, while not quantitatively accurate, may be assumed to be representative of general trends of temperature as a function of other variables in the simulation, and is used in that sense in the following discussion.

Coupled electro-thermal (ET) with radiation (R) modeling (ET\&R)was performed of the Wolfspeed JBS diode. This model solves for the lattice temperature by using the heat conduction equation. The model accounts for self-heating effects (via, e.g., Joule heating and carrier recombination) in semiconductor devices self-consistently with the TCAD transport equations. $\mathrm{SiC}$ offers a large thermal conductivity (of $\sim 350 \mathrm{~W} /(\mathrm{mK})$ at room temperature), which makes its use for power electronics very advantageous compared to other materials. However, the thermal conductivity coefficient $(k)$ decreases with temperature, while the specific heat coefficient $\left(C_{p}\right)$ increases with temperature. For the simulations presented here, we assumed a constant specific heat with temperature $\left(C_{p}\right.$ $=700 \mathrm{~J} /(\mathrm{kg} \mathrm{K}))$. A parametric fit of the thermal conductivity from [26] is: $k(T)=611 /(T-115) \mathrm{W} /(\mathrm{cm}-\mathrm{K})$, which was used in the present simulations. This temperature dependence results in even more localized lattice heating compared to a simulation with a constant room-temperature value of $k$, due to greatly reduced heat conduction at higher temperatures. The importance of a temperature dependent $k$ for accurate electrothermal modeling was also emphasized in [8] for a conventional SBD.

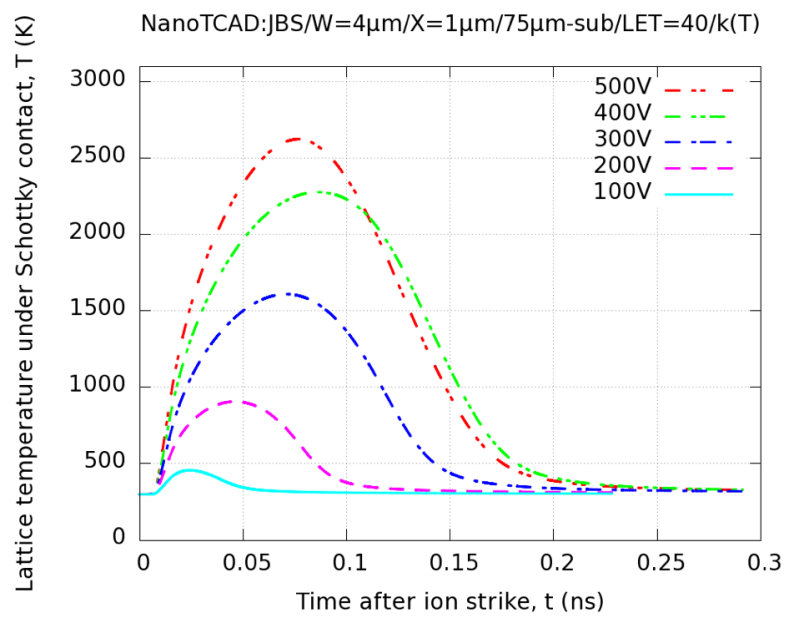

Fig. 11. Simulated temperature contours in the JBS diode in the semiconductor just below the Schottky contact showing Xe-ion induced localized heating profile. The contact temperature is held to $300{ }^{\circ} \mathrm{K}$ (perfect heat sink).

Figure 10 shows that for a Xe ion strike located midway between the P-wells, the local temperature increases significantly. The local temperature has a Gaussian profile, with a maximum of $\sim 3600 \mathrm{~K}$ (a value higher than the $\mathrm{SiC}$ melting temperature) at the center position of the ion strike. The lattice temperature remains above the melting temperature only within a range of 20-100 nm under the Schottky contact, and reaches this maximum within $\sim 100 \mathrm{ps}$ of the ion strike, in agreement with prior results $[1,7,8]$. A second temperature spike occurs at the epi-substrate interface, but this is much weaker.

The steps in the degradation of reverse leakage current (Fig. 3) may also be a result of a field/temperature effect. Silicon carbide is chemically unstable at temperatures $>1073 \mathrm{~K}(800 \mathrm{C})$ in the presence of most metals (cobalt, nickel, iron, etc.). The instability results from reactions of the metal with $\mathrm{SiC}$ to produce silicides and free carbon [27]. Precise onset of the mixing of the Schottky metal and the $\mathrm{SiC}$ is unknown, but it is reasonable to assume that leakage current degradation due to a eutectic-like mix of silicon carbide and the Schottky metal can occur above approximately $1000 \mathrm{~K}$.

Simulations of temperature and electric field as a function of time after the ion strike at a location just under the Schottky contact are shown in Figs. 11 and 12, parameterized by reverse bias voltage. Recognizing the caveats about simulation of temperature earlier stated, it appears that the temperature profile supports the degradation and burnout hypothesis. These simulations were performed assuming a contact temperature of $300 \mathrm{~K}$, which would be the case if a very efficient heatsink were attached to the device, so these estimates of temperature are actually conservative. At low bias voltages, such as $100 \mathrm{~V}$, the local temperature is safely within the normal operational range of crystalline silicon carbide. At $200 \mathrm{~V}$, the temperature approaches $1000 \mathrm{~K}$, where the eutectic interaction occurs, so the boundary between non-degraded and degraded leakage current behavior (such as that shown in Fig. 3) would be somewhere between 200 and $300 \mathrm{~V}$ bias. The $500 \mathrm{~V}$ 


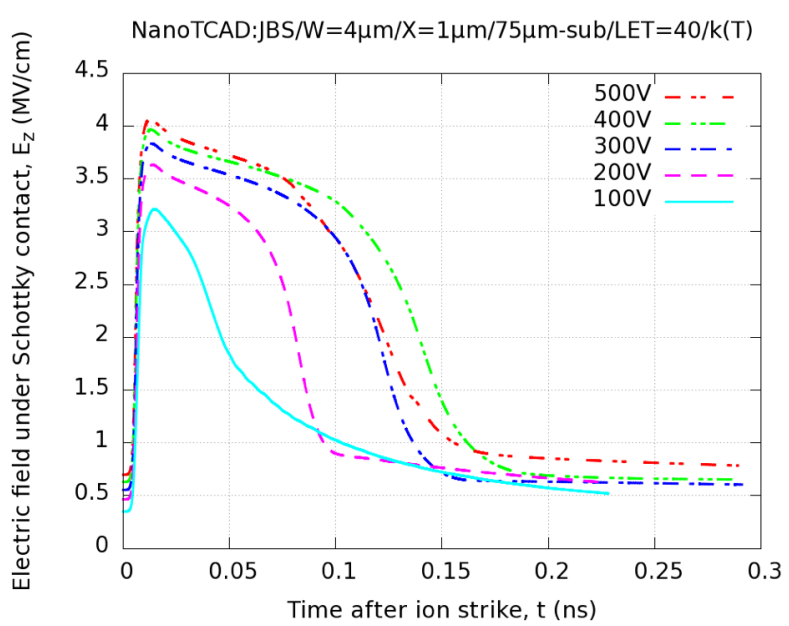

Fig. 12. Simulated vertical electric field contours in the JBS diode in the semiconductor just below the Schottky contact showing Xe-ion induced localized heating profile. The contact temperature is held to $300{ }^{\circ} \mathrm{K}$ (perfect heat sink).

temperature peak does not quite reach $3100 \mathrm{~K}$ (again these temperatures are conservative), but this supports the case that melting of the silicon carbide would be observed around $500 \mathrm{~V}$, resulting in a burnout event. Recent failure analysis results on silicon Schottky diodes indicates that devices that display degraded leakage current after exposure to heavy ions are found to have small regions of fused silicon under the Schottky junction, which may support the hypothesis that a phase change or eutectic interaction is causing the degraded behavior in silicon carbide JBS diodes [28].

The peak electric field profiles shown in Fig. 12 do not vary nearly as much as temperature does as a function of bias voltage, going from $3.2 \mathrm{MV} / \mathrm{cm}$ to $4.1 \mathrm{MV} / \mathrm{cm}$ over the whole range of reverse bias. However, the duration of the high electric field after a strike does increase significantly for biases of 200 $\mathrm{V}$ and above. Persistence of the high electric field may be necessary to reach the high peak temperatures shown in Fig. 11. The peak electric field saturates as a function of reverse bias, which may explain the relative insensitivity of the experimental boundaries in Fig. 6 to reverse bias above a certain LET.

\section{CONCLUSION}

In this work, data on single-event degradation and catastrophic failure of reverse biased $1200 \mathrm{~V}, 20 \mathrm{~A} \mathrm{SiC} \mathrm{Junction}$ Barrier Schottky (JBS) silicon carbide diodes are presented. The threshold for leakage degradation and the threshold for SEB are mapped as functions of bias and LET. Single-event induced reverse leakage current is observed at LETs as low as $3 \mathrm{MeV}^{*} \mathrm{~cm}^{2} / \mathrm{mg}$ at reverse voltages below device specifications. Above $10 \mathrm{MeV}^{*} \mathrm{~cm}^{2} / \mathrm{mg}$, burnout occurs at reverse voltages that are somewhat less than $50 \%$ of device specifications.
We suggest that onset of the degradation of the leakage current is caused by ions crossing the metal-semiconductor boundary of the Schottky diode and generating local temperatures that reach the regime where $\mathrm{SiC}$ interacts with the contact metal. We attribute the burnout phenomena to the semiconductor ion strike local temperature exceeding the melting point of the silicon carbide semiconductor. Electrothermal TCAD simulations are presented that are consistent with the experimental results and offer insights into possible mechanisms for degradation and burnout behavior. The results presented in this work are useful for survivability estimates for SiC JBS power diodes operating in harsh radiation environments and for gaining a better understanding of the basic mechanisms involved in the radiation response of this class of device.

\section{ACKNOWLEDGEMENT}

The authors thank Dr. J-M Lauenstein for useful discussions.

\section{REFERENCES}

[1] S. Kuboyama, C. Kamezawa, N. Ikeda, T. Hirao, and H. Ohyama,"Anomalous Charge Collection in Silicon Carbide Schottky Barrier Diodes and Resulting Permanent Damage and Single-Event Burnout," IEEE Trans. Nucl. Sci., vol. 53, no. 6, pp. 3343-3348, Dec. 2006.

[2] L. Scheick, L. Selva, and H. Becker, "Displacement damageinduced catastrophic second breakdown in silicon carbide Schottky power diodes," IEEE Trans. Nucl. Sci., vol. 51, no. 6, pp. 3193-3200, Dec. 2004.

[3] S. Kuboyama, C. Kamezawa, Y. Satoh, T. Hirao, and H. Ohyama, "Single-Event Burnout of Silicon Carbide Schottky Barrier Diodes Caused by High Energy Protons," IEEE Trans. Nucl. Sci., vol. 54, no. 6, pp. 2370-2383, Dec. 2007.

[4] M. C. Casey, J. Lauenstein, A. D. Topper, E. P. Wilcox, H. Kim, A. M. Phan, and K. A. Label, "Single-Event Effects in Silicon Carbide Power Devices," NASA NEPP Electronic Technology Workshop, June 2013, nepp.nasa.gov.

[5] J-M Lauenstein, M.C. Casey, and K. A. LaBel, "Single-Event Effects in Silicon and Silicon Carbide Power Devices," NASA NEPP Electronic Technology Workshop, June 2014, nepp.nasa.gov.

[6] E. Mizuta, S. Kuboyama, H. Abe, Y. Iwata, and T. Tamura, "Investigation of Single-Event Damages on Silicon Carbide (SiC) Power MOSFETs," IEEE Trans. Nucl. Sci., vol. 61, no. 4, pp. 1924-1928, Aug. 2014.

[7] C. Abbate et al., "Thermal damage in SiC Schottky diodes induced by SE heavy ions," Microelectron. Reliab., vol. 54, no. 9-10, pp. 2200-2206, 2014.

[8] C. Abbate et al., "Analysis of Heavy Ion Irradiation Induced Thermal Damage in SiC Schottky Diodes," IEEE Trans. Nucl. Sci., vol. 62, no. 1, pp. 202-209, Feb. 2015.

[9] A. Javanainen et al., "Charge Transport Mechanisms in HeavyIon Driven Leakage Current in Silicon Carbide Schottky Power Diodes," IEEE Trans. Device Mater. Reliab., vol. 16, no. 2, pp. 208-212, Jun. 2016.

[10] A. Javanainen et al.,"Heavy Ion Induced Degradation in $\mathrm{SiC}$ Schottky Diodes: Bias and Energy Deposition Dependence," IEEE Trans. Nucl. Sci., vol. 64, no. 1, pp. 415-420, Aug. 2017.

[11] A. Javanainen, et al.,"Heavy Ion Induced Degradation in $\mathrm{SiC}$ Schottky Diodes: Incident Angle and Energy Deposition Dependence," IEEE Trans. Nucl. Sci., vol. 64, no. 8, pp. 20312037, Aug. 2017. 
[12] T. Barbieri, "SiC Schottky Diode Device Design: Characterizing Performance \& Reliability," Technical Brief, www.wolfspeed.com

[13] N. Yang, "Characterization and modeling of silicon and silicon carbide power devices," PhD Dissertation, Virginia Polytechnic Institute and State University, 2010.

[14] A. Agarwal and S.-H. Ryu, "Status of SiC Power Devices and Manufacturing Issues," CS MANTECH Tech. Digest, pp. 215218, Vancouver, British Columbia, Canada, April 2006.

[15] N. Ren, J. Wang, K. Sheng, "Design and experimental study of 4H-SiC trenched junction barrier Schottky diodes," IEEE Trans. Electron Dev., vol. 61, no. 7, pp. 2459-2465, Jul. 2014.

[16] N. Ren and K. Sheng, "An Analytical Model With 2-D Effects for 4H-SiC Trenched Junction Barrier Schottky Diodes," IEEE Trans. Electron Dev., vol. 61, no. 12, pp. 4158-4165, Dec. 2014.

[17] Feng-Ping Chen, Yu-Ming Zhang, Yi-Mien Zhang, and Jian-Hya Huang, "Study of $4 \mathrm{H}-\mathrm{SiC}$ junction barrier Schottky diode using field guard ring termination," Chinese Phys. B, vol. 19, pp. 097$107,2010$.

[18] A. M. Albadri, R.D. Schrimpf, D.G. Walker, and A.V. Mahajan, "Coupled Electro-Thermal Simulations of Single Event Burnout in Power Diodes," IEEE Trans. Nucl. Sci., vol. 52, no. 6, pp. 2194-2199, Dec. 2005.

[19] V. Uhnevionak, Simulation and Modeling of Silicon-Carbide Devices, PhD Dissertation, University of Erlangen-Nuremberg, October 2015.

[20] X. Zhang, Failure Mechanisms in Wideband Semiconductor Power Devices, PhD Dissertation, University of Maryland, College Park, 2006.

[21] Ioffe: http://www.ioffe.rssi.ru/SVA/NSM/Semicond/SiC/

[22] M. Roschke and F. Schwierz, "Electron Mobility Models for 4H, 6H, and 3C SiC", IEEE Trans. Elec. Dev., Vol. 48, No. 7, pp. 1442-1447, Jul. 2001.

[23] L. Zhu and T. P. Chow, "Analytical Modeling of High-Voltage 4H-SiC Junction Barrier Schottky (JBS) Rectifiers," IEEE Transactions on Electron Devices, vol. 55, no. 8, pp. 1857-1863, Aug. 2008.

[24] S. Kuboyama, C. Kamezawa, N. Ikeda, T. Hirao, and H. Ohyama, "Anomalous Charge Collection in Silicon Carbide Schottky Barrier Diodes and Resulting Permanent Damage and SingleEvent Burnout," IEEE Trans. Nucl. Sci., vol. 53, no. 6, pp. 33433348, Dec. 2006.

[25] M. Roschke and F. Schwierz, "Electron mobility models for 4H, 6H, and 3C SiC [MESFETs]," IEEE Trans. Electron Dev., vol. 48, no. 7, pp. 1442-1447, Jul. 2001.

[26] Y. Goldberg, M.E. Levinshtein, S.L Rumyantsev. Properties of Advanced Semiconductor Materials GaN, AlN, SiC, BN, SiC, SiGe, John Wiley \& Sons, Inc., New York, 2001, pp. 93-148.

[27] Y. and J.L. Baptista, "Chemical Instability of Silicon Carbide in the Presence of Transition Metals," J. Am Ceram. Soc. Vol. 79, no. 8, pp. 2017-2026, 1996.

[28] M. C. Casey, J.-M. Lauenstein, E.P. Wilcox, A. D. Topper, R. L. Ladbury, M. J. Campola, K.A. LaBel, "Failure Analysis of Heavy-Ion Irradiated Schottky Diodes," IEEE Trans. Nucl. Sci., accepted for Jan. 2018. 